\title{
Mosaic trisomy 15
}

INSERM

\section{Source}

INSERM. (1999). Orphanet: an online rare disease and orphan drug data base. Mosaic trisomy 15. ORPHA:1706

Mosaic trisomy 15 is a rare chromosomal anomaly syndrome principally characterized by intrauterine growth restriction, congenital cardiac anomalies (incl. ventricular and atrial septal defects, patent ductus arteriosus) and craniofacial dysmorphism (incl.

hypertelorism, downslanting palpebral fissures, wide nasal bridge). Patients also present brain (e.g. hypoplastic cerebellum, ventricular asymmetry), renal (e.g. small dysplastic kidneys), and/or genital (undescended testis, small penis, hypoplastic labia majora) anomalies. Digital and skin pigmentation abnormalities have also been reported. 